\title{
Transcutaneous electrical acupoint stimulation for stage 1 hypertension: protocol for a randomized controlled pilot trial
}

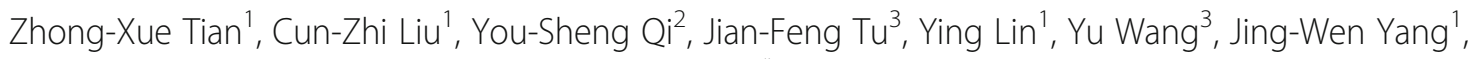
Guang-Xia Shi ${ }^{1}$, Jun-Hong Liu ${ }^{2}$ and Li-Qiong Wang ${ }^{1 *}$

\begin{abstract}
Background: Hypertension is a major pathogenic factor of cardiovascular diseases. Insufficient blood pressure control rate and sub-optimal medication adherence remain challenges for effective management of hypertension.

Transcutaneous electrical acupoint stimulation (TEAS) has been used to treat various diseases, including hypertension, but the scientific evidence for its benefit remains insufficient. Therefore, we will perform a randomized, controlled clinical trial in patients with stage 1 hypertension to evaluate the effect of TEAS.

Methods/design: The study will be a two-arm parallel, randomized controlled trial. Sixty patients with stage 1 hypertension will be randomly assigned to the TEAS group and the control group in a 1:1 ratio. The participants in the TEAS group will receive non-invasive acupoint electrical stimulation for $30 \mathrm{~min}$ at four acupoints in the upper and lower extremities at home, 4 times weekly for 12 weeks for a total of 48 sessions. Participants in the control group will not receive any form of acupoint stimulation. All participants in both groups will receive lifestyle education on how to control high blood pressure, including diet, weight control, and exercise. The primary outcome measure will be the change of the mean systolic blood pressure from baseline to 12 weeks. Secondary outcomes include the change of mean diastolic blood pressure, quality of life, body mass index, and physical activity level.
\end{abstract}

Discussion: This pilot, randomized, controlled trial will explore the feasibility of TEAS. It will also provide potential clinical evidence for the efficacy and safety of TEAS in the treatment of patients with stage 1 hypertension. The results of this study will be published in peer-reviewed journals. Furthermore, this pilot trial as the precursor of a large scale randomized controlled trial will inform the sample size of the subsequent trial.

Trial registration: Chinese clinical trial registry, ChiCTR1900025042, Registered on 8 August 2019 (http://www. chictr.org.cn/showproj.aspx?proj=41496).

Keywords: Transcutaneous electrical acupoint stimulation (TEAS), Lifestyle education, Stage 1 hypertension, Randomized controlled trial

\footnotetext{
* Correspondence: wangliqiongwork@163.com

'Acupuncture Research Center, School of Acupuncture-Moxibustion and

Tuina, Beijing University of Chinese Medicine, No. 11, Bei San Huan Dong Lu,

Chaoyang District, Beijing 100029, China

Full list of author information is available at the end of the article
}

(c) The Author(s). 2020 Open Access This article is licensed under a Creative Commons Attribution 4.0 International License, which permits use, sharing, adaptation, distribution and reproduction in any medium or format, as long as you give appropriate credit to the original author(s) and the source, provide a link to the Creative Commons licence, and indicate if changes were made. The images or other third party material in this article are included in the article's Creative Commons. licence, unless indicated otherwise in a credit line to the material. If material is not included in the article's Creative Commons licence and your intended use is not permitted by statutory regulation or exceeds the permitted use, you will need to obtain permission directly from the copyright holder. To view a copy of this licence, visit http://creativecommons.org/licenses/by/4.0/ The Creative Commons Public Domain Dedication waiver (http://creativecommons.org/publicdomain/zero/1.0/) applies to the data made available in this article, unless otherwise stated in a credit line to the data. 


\section{Background}

Hypertension is a major risk factor for cardiovascular disease death and its global burden is increasing [1]. Stage 1 hypertension is a systolic blood pressure (SBP) of $140-159 \mathrm{mmHg}$ or diastolic blood pressure (DBP) of $90-99 \mathrm{mmHg}$ and is one of the common subtypes of hypertension in China [2]. Previous studies have shown that a $10-\mathrm{mmHg}$ reduction in SBP or a $5-\mathrm{mmHg}$ reduction in DBP significantly reduces the relative risk of coronary heart disease [3], and blood pressure (BP) control in patients with stage 1 hypertension below $140 / 90 \mathrm{mmHg}$ also significantly reduced the risk of stroke or death [4]. Therefore, it is necessary to the adequately control BP in patients with stage 1 hypertension.

At present, the treatment of hypertension includes the use of antihypertensive drugs and lifestyle interventions [5]. A systematic review showed that drug treatment is ineffective for many patients with stage 1 hypertension [6]. Therefore, widely used lifestyle changes, including weight loss, reduced sodium intake, regular physical exercise, smoking cessation, and moderate alcohol consumption, are also important [7, 8]. However, the benefits of lifestyle interventions on blood pressure will decrease over time $[9,10]$. Therefore, a sustainable and effective long-term non-drug treatment is needed [11].

Acupuncture is an important part of traditional Chinese medicine therapy and has proven antihypertensive effect in hypertensive patients [12-15]. However, some patients worry about the economic cost of acupuncture or the time it takes to get to the hospital to have it [16, 17]. Transcutaneous electrical acupoint stimulation (TEAS) is a new non-invasive acupuncture treatment, which combines the effects of transcutaneous electrical nerve stimulation (TENS) with acupoint stimulation [18]. It stimulates the afferent nerves with low-voltage pulses transmitted through the skin at an acupoint, and TEAS may have a better antihypertensive effect than traditional acupuncture. In a study, Jacobsson et al. found that TEAS may have additional antihypertensive effect on 24-h ambulatory BP in those patients who did not respond to pharmacological treatment [19]. But it had some limitations, including the low quality of the study design and inadequate control groups. There are no randomized controlled trials to evaluate the efficacy of TEAS in the treatment of stage 1 hypertension at the present time.

In this study, we hypothesize that TEAS has an additional antihypertensive effect on stage 1 hypertension patients after lifestyle interventions. The results derived from this study will be used to explore the efficacy and safety of TEAS in lowering BP and calculated the appropriate sample size for a future large clinical trial.

\section{Methods/design \\ Study design}

This open-label, two-armed, randomized, controlled pilot trial will be conducted at Nanyuan Community Health Service Center which is one of chronic disease management bases in Beijing, China. The protocol has been registered on Chinese clinical trial registry (No ChiCTR1900025042) and approved by the ethics committee of Beijing University of Chinese Medicine (No 2019BZHYLL0208). The protocol follows the Declaration of Helsinki and will be reported in accordance with the Standard Protocol Items (SPIRIT) (Additional file 1).

\section{Study setting, recruitment}

The clinical research coordinator will recruit 60 patients with stage 1 hypertension at Nanyuan Community Health Service Center in Beijing. The main recruitment strategies include the community's chronic disease management software, WeChat advertisements, and on-site flyers at the outpatient department. The clinical research coordinator will conduct preliminary information screening by telephone or face to face. Then, patients who pass the preliminary screening will undergo a blood pressure test to determine whether they will be finally included. Written informed consent will be obtained by the clinical research coordinator at the outpatient department before randomization. Figure 1 shows the flow diagram of the trial.

\section{Participants \\ Inclusion criteria}

1. Aged between 35 and 65 years (male or female)

2. Fulfilling the diagnostic criteria for stage 1 hypertension (SBP of 140-159 $\mathrm{mmHg}$ and/or DBP of $90-99 \mathrm{mmHg}$ ) [20]

3. Capacity to consent to taking part in the study and complete the questionnaires.

4. Voluntary signing of informed consent

\section{Exclusion criteria}

1. Contraindications for the use of acupoint stimulators: wearing a pacemaker and other implanted medical devices; suffering from acute diseases, infectious diseases, malignant tumors; cardiovascular disease, cerebrovascular disease, liver and kidney dysfunction, or other malignant diseases; scars, bruises, scratches, or inflammation on the skin of the acupuncture points.

2. Secondary hypertension caused by other vascular diseases, primary aldosteronism, other endocrine hypertension, etc. 


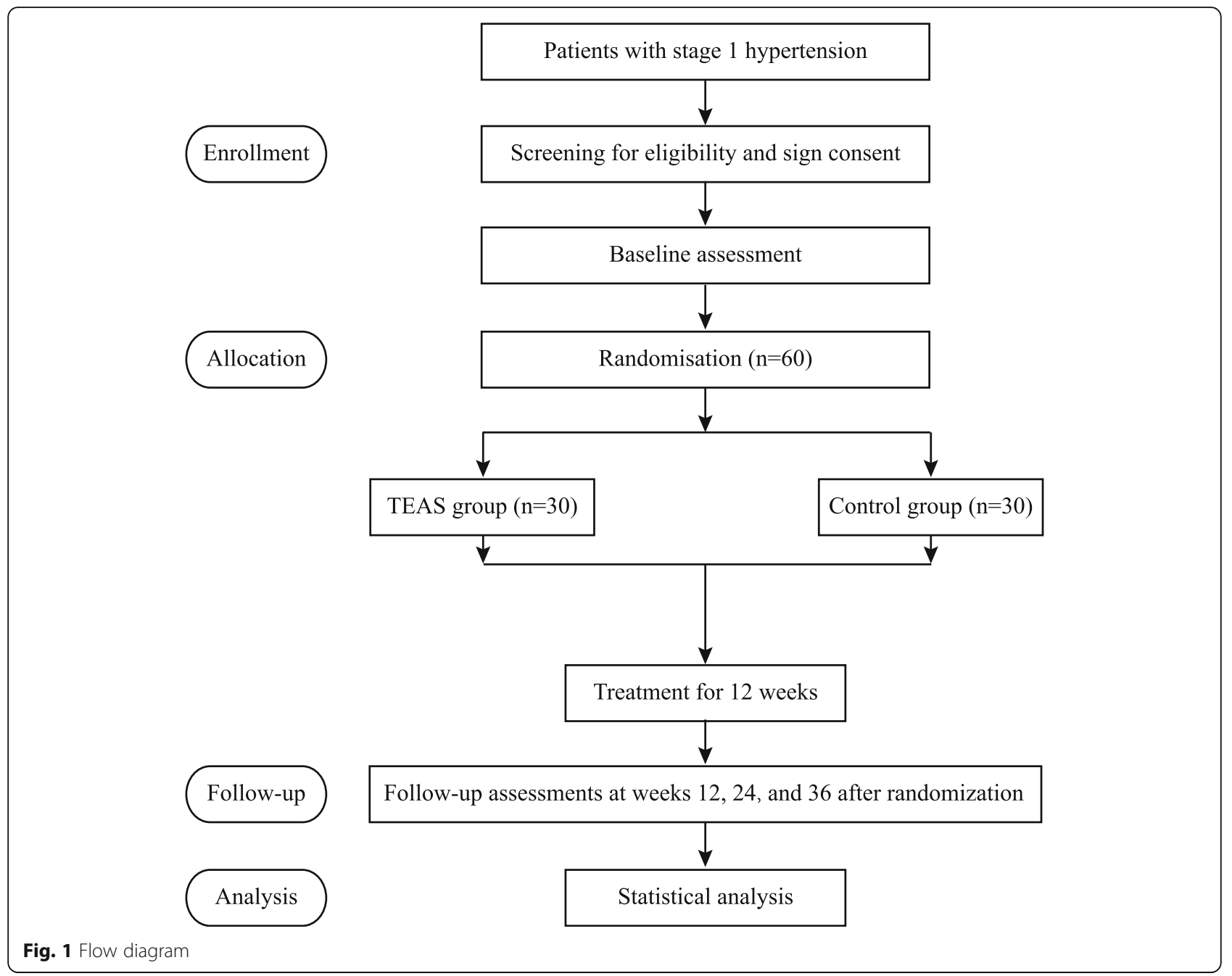

3. Receiving drugs that affect blood pressure except antihypertensive drugs in previous 2 months such as glucocorticoids, central nervous system inhibitors.

4. Uncontrolled diabetes.

5. Drug or alcohol abuse.

6. Pregnant women or women in lactation period.

7. Acupuncture treatment within the past month.

8. Participation in another clinical study in the past month.

\section{Randomization and masking}

Eligible patients will be randomly assigned to the TEAS group or control group in a ratio of 1:1. The randomization sequence will be generated with the software SAS 9.3 by an independent statistician (LQ. Wang, Beijing University of Chinese Medicine, China) using block randomization. Randomization sequence will be stored by a non-involved investigator. When an eligible patient needs to be randomized, the clinical research coordinator will contact the non-involved investigator by phone to obtain random number and assign him/her to one group. The design is open label, but the outcome assessors and statisticians who are blinded to group allocation will be responsible for collecting and analyzing the data, respectively.

\section{Interventions}

The physician will provide lifestyle education to all patients for 12 weeks, which mainly includes sending relevant antihypertensive information to patients every week and holding health lectures every month. The content of the information includes influence of lifestyle on hypertension control, especially the strength of dietary health, and the importance of moderate physical exercise, maintaining normal weight and quitting smoking. In addition, we will establish a communication group to answer questions raised by patients during the intervention. If patients were on antihypertensive medication, they were instructed to continue this medication but to abstain from changing the type or dosage of drugs. 


\section{Control group}

Subjects randomized to the control group will not be given TEAS treatment except the lifestyle education.

\section{Transcutaneous electrical acupoint stimulation group}

In addition to the lifestyle education, the participants in this group will receive TEAS 48 sessions over a period of 12 weeks (4 sessions/week) at home. TEAS will be performed using a portable instrument for low-frequency electrotherapy (SDP-330, Yuwell, Suzhou Medical Appliances Co, Ltd., Suzhou, China). The device is a so-called dual channel stimulator with two pairs of rubber electrodes $(30 \mathrm{~mm} \times 30 \mathrm{~mm})$ applied to the skin as illustrated in Fig. 2. The instrument has 8 different stimulus modes; patients will be asked to select a fixed mode ("press" or "knock" or "knead" mode) throughout the trial. It has ten different stimulus intensities, and patients could choose based on their tolerance.

According to the previous studies [21, 22], bilateral Hegu (LI4), Quchi (LI11), Zusanli (ST36), and Taichong (LR3) will be selected in our trial. At the first session, the electrodes will be placed at the ipsilateral LI4 and LI11, and then the appropriate stimulus intensity will be selected according to tolerance of each individual for 15 min after connecting the instrument, then the opposite limb will be treated in the same way for $15 \mathrm{~min}$. In the second session, the ST36 and LR3 acupoints of the leg will be treated for $15 \mathrm{~min}$ in the same way. Two pairs of acupoints will be stimulated alternatively every other day. Locations of acupoints are shown in Table 1.

The acupuncturist will train the participants on how to locate the acupoints and use the instrument face-toface. They will also send paper instructions and acupoint maps to help patients with TEAS treatment at home. The patients will be required to record the date, the time, and the specific acupoints when using the instrument. The instrument will be given to the patients when

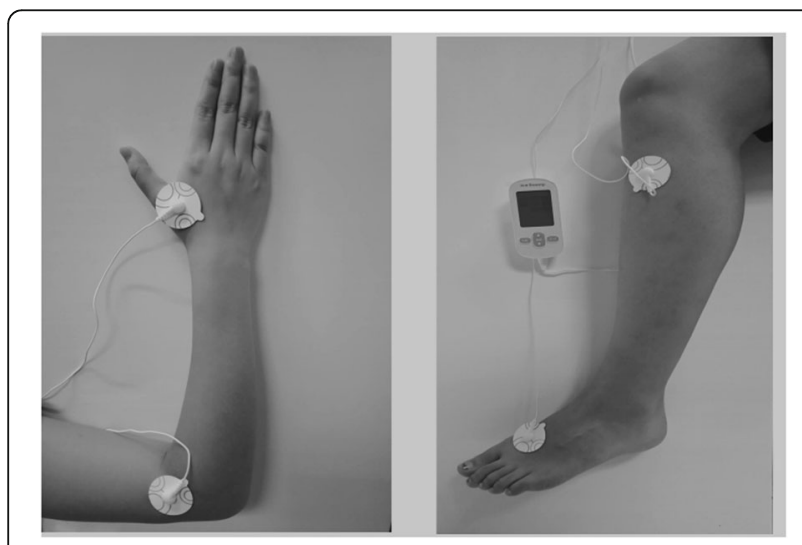

Fig. 2 Locations of acupoints
Table 1 Locations of acupoints in the TEAS group

\begin{tabular}{|c|c|}
\hline Acupoints & Locations $^{a}$ \\
\hline Hegu (LI4) & $\begin{array}{l}\text { On the dorsum of the hand, radial to the midpoint of the } \\
\text { second metacarpal bone. }\end{array}$ \\
\hline Quchi (LI11) & $\begin{array}{l}\text { On the lateral aspect of the elbow, at the midpoint of the } \\
\text { line connecting } \\
\text { LU5 with the lateral epicondyle of the humerus. }\end{array}$ \\
\hline $\begin{array}{l}\text { Zusanli } \\
\text { (ST36) }\end{array}$ & $\begin{array}{l}\text { On the anterior aspect of the leg, on the line connecting } \\
\text { ST35 with ST41, } 3 \text { B-cun inferior to ST35. ST36 is located } \\
\text { on the tibialis anterior muscle. }\end{array}$ \\
\hline $\begin{array}{l}\text { Taichong } \\
(\text { LR3) }\end{array}$ & $\begin{array}{l}\text { In the depression anterior to the junction of first and } \\
\text { second metatarsal bones. }\end{array}$ \\
\hline
\end{tabular}

they have completed the prescribed treatment times and visits.

\section{Outcomes}

\section{Primary outcome}

The primary outcome of the study is the change in mean SBP between baseline and 12 weeks after treatment. BP will be measured and recorded by using an electronic sphygmomanometer (HEM-7136, Omron Corporation, Kyoto, Japan) in both groups. Patients were asked to rest quietly for at least $5 \mathrm{~min}$ before taking blood pressure measurements of the upper arm in the sitting position, with the upper arm at heart level [23]. Blood pressure in both upper arms should be measured at the first test; the side with the higher blood pressure reading will be selected. At subsequent detection time points, the measurement will be repeated 3 times every $5 \mathrm{~min}$; the average value of the last 2 readings will be taken as the mean BP [24].

\section{Secondary outcomes}

The mean SBP will be evaluated at weeks 24 and 36 after randomization. The mean DBP, body mass index (BMI), International Sports Activity Questionnaire (IPAQ), and Short Form (SF-12) will be evaluated at baseline, 12 weeks, 24 weeks, and 36 weeks. The DBP measurement method is consistent with SBP. BMI is weight $(\mathrm{kg})$ divided by the square of height $[25,26]$. The IPAQ questionnaire includes the frequency, intensity, timing, and type of exercise [27], which will be divided into three levels of physical activity levels by metabolic equivalent (MET). The SF-12 is a questionnaire to evaluate general health outcomes including psychological and physical domains $[28,29]$.

The schedule of enrolment, intervention, and assessments is shown in Fig. 3.

\section{Adverse events}

Any adverse events will be reported by the patients and outcome assessors, including severe pain, local infection, 


\begin{tabular}{|c|c|c|c|c|c|}
\hline & \multicolumn{5}{|c|}{ STUDY PERIOD } \\
\hline \multirow[b]{2}{*}{ TIMEPOINT } & \multirow{2}{*}{$\begin{array}{c}\text { Enrolment } \\
\text { Week -1 }\end{array}$} & \multirow{2}{*}{$\begin{array}{c}\text { Allocation } \\
\text { Week } 0\end{array}$} & \multicolumn{2}{|c|}{ Post-allocation } & \multirow{2}{*}{$\begin{array}{l}\text { Closeout } \\
\text { Week } 36\end{array}$} \\
\hline & & & Week 12 & Week 24 & \\
\hline \multicolumn{6}{|l|}{ ENROLMENT: } \\
\hline \multirow{4}{*}{$\begin{array}{r}\text { Eligibility screen } \\
\text { Informed consent } \\
\text { Randomization } \\
\text { Allocation }\end{array}$} & $x$ & & & & \\
\hline & $x$ & & & & \\
\hline & $x$ & & & & \\
\hline & & $x$ & & & \\
\hline \multicolumn{6}{|l|}{ INTERVENTIONS: } \\
\hline \multirow{2}{*}{$\begin{array}{r}\text { TEAS intervention } \\
\text { Control group }\end{array}$} & & $\leftarrow$ & $\longrightarrow$ & & \\
\hline & & $\leftarrow$ & $\longrightarrow$ & & \\
\hline \multicolumn{6}{|l|}{ ASSESSMENTS: } \\
\hline SBP & & $\times$ & $x$ & $x$ & $x$ \\
\hline DBP & & $x$ & $x$ & $x$ & $x$ \\
\hline BMI & & $x$ & $x$ & $x$ & $x$ \\
\hline IPAQ & & $x$ & $x$ & $x$ & $x$ \\
\hline SF-12 & & $x$ & $x$ & $x$ & $x$ \\
\hline Safety assessment & & $x$ & $x$ & $x$ & $x$ \\
\hline
\end{tabular}

Fig. 3 Schedule of enrolment, intervention, and assessments (SPIRIT) figure

or unbearable tingling during treatment, and will be recorded during the trial. If any serious adverse events or uncontrolled hypertension occur, they will be immediately reported to the primary investigator and the patient will be withdrawn from the trial. There is no anticipated harm and compensation for trial participation.

\section{Quality control}

The program has been reviewed and revised by clinical acupuncturists and statisticians. Prescribed standard operating procedures are screening of patients, blood pressure monitoring, therapeutic criteria, random methods, and acupoint stimulation, and the case report form (CRF) will be filled in. The CRF will be completed on paper copies and then entered into the Excel spreadsheet to ensure the accuracy of the data. All modifications of the data can be traced through the CRF. The ethics committee of Beijing University of Chinese medicine will audit trial conduct per 12 months.

\section{Sample size}

This is a pilot trial, and the sample size is based on the minimum sample size of clinical trials. Each group will include 30 participants, a total of 60 participants. This is the minimum number of participants used for comparative analysis to determine the initial data of main outcome measurements and explore for future large-scale randomized controlled trial [30].

\section{Statistical analysis}

Statistical analysis will be conducted by SPSS version 23.0 (IBM SPSS Statistics, NY, USA). Intention-to-treat analysis (ITT) will be applied to all cases randomly assigned to each group [31]. We will try our best to collect the data for the patients who quit or lost the visit in this trial. The last observation carried forward (LOCF) will be used for missing data. There is no interim analysis or additional analysis in this trial. The level of significance will be established at $\alpha<0.05$ with a two-sided test. Patients' baseline characteristics will be described for the two groups. Continuously distributed variables will be described using mean \pm standard deviation $(\mathrm{M} \pm$ $\mathrm{SD})$ or median and quartile intervals. Discrete variables will be described by frequencies and percentages. For the primary outcome, the change in SBP between baseline and 12 weeks after treatment will be calculated and the difference of the change in SBP between the two groups will be tested using Student's $t$ test or the Wilcoxon rank sum test. For secondary outcomes, Student's $t$ test, chi-squared test, Fisher's exact test, or the Wilcoxon rank sum test will be used to test the difference of the outcomes including the DBP, BMI, IPAQ, and SF12 , between groups according to the distribution of variables.

\section{Discussion}

The awareness, treatment, and control of hypertension are insufficient in China [32]. For early hypertension, the 
symptoms are inconspicuous so that most patients pay little attention to the condition and may ultimately develop severe hypertension, thus increasing the risk of cardiovascular diseases [33]. In addition to the financial burden, drug treatment has potential side effects [34]. For patients with high blood pressure who refuse to take medicine or have low drug compliance, other effective methods of lowering blood pressure need to be found.

It has been shown that acupuncture is an effective way to lower blood pressure [35, 36]. As a non-invasive treatment, TEAS is similar to acupuncture in stimulating acupoints, and many clinical studies have shown the feasibility and safety of TEAS [37-39]. This approach to intervention has several advantages. First, the selfadjusting mode of stimulation parameters allows patients to adjust the frequency and intensity of treatment according to their own endurance capacity to prevent skin damage or other adverse reactions. Second, patients can use it at home at any time, which greatly saves time and economic cost. The study will be conducted within 12 weeks to observe the long-term effects of TEAS on patients with stage 1 hypertension.

In addition to feasible interventions, the use of an appropriate control group is a key issue in designing clinical trials. In mild hypertension, lifestyle change is a necessary step to lower blood pressure [40]. We will test whether TEAS combined with lifestyle education has a greater effect on lowering blood pressure and controlling conditions in patients with stage 1 hypertension. To minimize the confounding effects, the two groups will receive scientific information about hypertension lifestyle interventions at the same time. During the trial, we will call or send messages to encourage patients in the TEAS group to adhere to the instrument treatment to improve compliance.

The limitation of our trial is that the patients are not blinded. Based on the nature of chronic disease and barrier of time necessary for frequent transportation to hospital, the majority of treatment will be operated by patients themselves at home. And patients can distinguish whether the instrument is on or not. It is difficult to blind patients. Moreover, this pilot trial is the precursor to a large scale randomized controlled trial in which the aim is to evaluate the effectiveness not the efficacy of transcutaneous electrical acupoint stimulation as adjunctive therapy for stage 1 hypertension in the real world. Hence, we do not set sham therapy as the control group.

In conclusion, we hypothesize that the systolic blood pressure in the TEAS group will be lower than that in the control group after 12 weeks of treatment, and the results will allow patients to delay the use of hypertensive drugs, prevent patients from becoming seriously hypertensive hypertension, and provide evidence for future large clinical trials. Our results will be published in peer review journals in the form of articles.

\section{Trial status}

Protocol: version 2.0, 10 June 2019.

The first patient was recruited on 4 September 2019. At the present time, a total of 30 patients had been randomized. The final date of follow-up is expected to be October 2020. This protocol was submitted prior to the recruitment of total 30 patients.

\section{Supplementary information}

Supplementary information accompanies this paper at https://doi.org/10. 1186/s13063-020-04493-x.

Additional file 1. Completed Standard Protocol Items:

Recommendation for Interventional Trials (SPIRIT) 2013 Checklist: items addressed in this clinical trial protocol.

\section{Abbreviations}

TEAS: Transcutaneous electrical acupoint stimulation; TENS: Transcutaneous electrical nerve stimulation; BP: Blood pressure; BMI: Body mass index; SBP: Systolic blood pressure; DBP: Diastolic blood pressure; CRF: Case report form; SF-12: 12-Item Short Form Health Survey; ITT: Intention-to-treat analysis; LOCF: Last observation carried forward

\section{Acknowledgements}

The authors would like to express their sincere thanks to all patients participating in the trial.

\section{Authors' contributions}

Liu CZ conceived of the study. Wang LQ initiated the study design. Tian ZX and Tu JF drafted this manuscript. Wang Y, Liu JH, and Qi YS drew up the statistical plan. Wang LQ, Tu JF, Ling Y, and Liu CZ drafted and helped with its implementation. All authors contributed to the refinement of the study protocol and approved the final manuscript.

\section{Funding}

This work was supported by the Distinguished Young Scholars Project of Beijing University of Chinese Medicine (BUCM-2019-JCRC011). The sponsor has no role in designing the trial, collection, management, analysis, and interpretation of data and the decision to write a report or submit a report for publication.

\section{Availability of data and materials}

All of the individual participant data collected during the trial after deidentification will be available for anyone who wishes to access the data immediately following publication. Any data required to support the protocol can be supplied on request.

\section{Ethics approval and consent to participate}

This trial has been approved by Beijing University of Chinese Medicine (2019BZHYLL0208). Written informed consent will be obtained from patients before randomization. If we have important changes to the protocol, the principal investigator will notify the center and that a copy of the revised protocol will be sent to the principal investigator to add to the Investigator Site File. What is more, we will also update the protocol in the clinical trial registry if there is any change. Any deviations from the protocol will be fully documented using a breach report form.

\section{Consent for publication}

Written informed consent for publication of Fig. 2 was obtained from the individual. This is available from the corresponding author on request. 


\section{Author details}

'Acupuncture Research Center, School of Acupuncture-Moxibustion and Tuina, Beijing University of Chinese Medicine, No. 11, Bei San Huan Dong Lu, Chaoyang District, Beijing 100029, China. ${ }^{2}$ Nanyuan Community Health Service Center, Fengtai District, Beijing, China. ${ }^{3}$ Department of Acupuncture and Moxibustion, Beijing Hospital of Traditional Chinese Medicine Affiliated to Capital Medical University, Dongcheng District, Beijing, China.

Received: 16 January 2020 Accepted: 10 June 2020

Published online: 22 June 2020

\section{References}

1. Lackland DT, Weber MA. Global burden of cardiovascular disease and stroke: hypertension at the core. Can J Cardiol. 2015;31:569-71.

2. Qi SF, Zhang B, Wang HJ, Yan J, Mi YJ, Liu DW, et al. Prevalence of hypertension subtypes in 2011 and the trends from 1991 to 2011 among Chinese adults. J Epidemiol Community Health. 2016;70:444-51.

3. Lewington S, Clarke R, Qizilbash N, Peto R, Collins R. Prospective Studies Collaboration. Age-specific relevance of usual blood pressure to vascular mortality: a meta-analysis of individual data for one million adults in 61 prospective studies. Lancet. 2002;360:1903-13.

4. Qin X, Li Y, Sun N, He M, Tang G, Yin D, et al. Impact of achieved blood pressure on first stroke in uncomplicated grade 1 hypertension. J Am Heart Assoc. 2017;6:e005247.

5. Williams B, Mancia G, Spiering W, Agabiti Rosei E, Azizi M, Burnier M, et al. 2018 Practice Guidelines for the management of arterial hypertension of the European Society of Hypertension and the European Society of Cardiology: ESH/ESC Task Force for the Management of Arterial Hypertension. J Hypertens. 2018;36:2284-309.

6. Lenzer J. Cochrane review finds no proved benefit in drug treatment for patients with mild hypertension. BMJ. 2012;345:e5511.

7. Dickinson HO, Mason JM, Nicolson DJ, Campbell F, Beyer FR, Cook JV, et al. Lifestyle interventions to reduce raised blood pressure: a systematic review of randomized controlled trials. J Hypertens. 2006;24:215-33.

8. Nicolson DJ, Dickinson HO, Campbell F, Mason JM. Lifestyle interventions or drugs for patients with essential hypertension: a systematic review. J Hypertens. 2004;22:2043-8.

9. Hinderliter AL, Sherwood A, Craighead LW, Lin PH, Watkins L, Babyak MA, et al. The long-term effects of lifestyle change on blood pressure: one-year follow-up of the ENCORE study. Am J Hypertens. 2014;27:734-41.

10. Baena CP, Olandoski M, Younge JO, Buitrago-Lopez A, Darweesh SK, Campos $\mathrm{N}$, et al. Effects of lifestyle-related interventions on blood pressure in low and middle-income countries: systematic review and meta-analysis. J Hypertens. 2014:32:961-73.

11. Espín-Sanchez R. Is there non-pharmacological treatment of hypertension? What is changed on hypertension treatment since Alderman in The Lancet 1994. Hipertens Riesgo Vasc. 2016;33:74-5.

12. Wang J, Xiong X, Liu W. Acupuncture for essential hypertension. Int J Cardiol. 2013;169:317-26.

13. Lee H, Kim SY, Park J, Kim YJ, Lee H, Park HJ. Acupuncture for lowering blood pressure: systematic review and meta-analysis. Am J Hypertens. 2009; 22:122-8.

14. Liu F, Guo C, Jin X. Acupuncture for mild-to-moderate essential hypertension: a meta-analysis of randomized clinical trials. Chin J Basic Med Tradit Chin Med. 2012:18:421-3.

15. Zhao XF, Hu HT, Li JS, Shang HC, Zheng HZ, Niu JF, et al. Is acupuncture effective for hypertension? A systematic review and meta-analysis. PLoS One. 2015;10:e0127019.

16. Wonderling D. The cost-effectiveness of acupuncture. Eur J Integr Med. 2015;7:437-8.

17. Kim SY, Lee $H$, Chae Y, Park HJ, Lee H. A systematic review of costeffectiveness analyses alongside randomised controlled trials of acupuncture. Acupunct Med. 2012;30:273-85.

18. Francis RP, Johnson MI. The characteristics of acupuncture-like transcutaneous electrical nerve stimulation (acupuncture-like TENS): a literature review. Acupunct Electrother Res. 2011:36:231-58.

19. Jacobsson F, Himmelmann A, Bergbrant A, Svensson A, Mannheimer C. The effect of transcutaneous electric nerve stimulation in patients with therapyresistant hypertension. J Hum Hypertens. 2000;14:795-8.

20. Joint Committee for Guideline Revision. 2018 Chinese guidelines for prevention and treatment of hypertension-a report of the revision committee of Chinese guidelines for prevention and treatment of hypertension. J Geriatr Cardiol. 2019;16:182-241.

21. Yang DH. Effect of electroacupuncture on Quchi (LI 11) and Taichong (LR 3) on blood pressure variability in young patients with hypertension. Zhongguo Zhen Jiu. 2010;30:547-50.

22. Yin C, Du YZ. Observation of anti-hypertensive effect on primary hypertension treated with acupuncture at Renying (ST 9) mainly. Zhongguo Zhen Jiu. 2012;32:776-8.

23. Climie RE, Schultz MG, Nikolic SB, Ahuja KD, Fell JW, Sharman JE. Validity and reliability of central blood pressure estimated by upper arm oscillometric cuff pressure. Am J Hypertens. 2012;25:414-20.

24. Shimamoto K, Ando K, Fujita T, Hasebe N, Higaki J, Horiuchi M, et al. The Japanese Society of Hypertension Guidelines for the Management of Hypertension (JSH 2014). Hypertens Res. 2014;37:253-390.

25. Eckel N, Li Y, Kuxhaus O, Stefan N, Hu FB, Schulze MB. Transition from metabolic healthy to unhealthy phenotypes and association with cardiovascular disease risk across BMI categories in 90257 women (the Nurses' Health Study): 30 year follow-up from a prospective cohort study. Lancet Diabetes Endocrinol. 2018;6:714-24.

26. Das RK, Nessa A. Blood pressure in different levels of BMI. Mymensingh Med J. 2013;22:699-705.

27. Hallal PC, Victora CG. Reliability and validity of the International Physical Activity Questionnaire (IPAQ). Med Sci Sports Exerc. 2004;36:556.

28. Ware J Jr, Kosinski M, Keller SD. A 12-Item Short-Form Health Survey: construction of scales and preliminary tests of reliability and validity. Med Care. 1996:34:220-33.

29. Wan E, Yu E, Chin WY, Choi E, Wu T, Lam C. Evaluation of the responsiveness of Short Form-12 Health Survey version 2 (SF-12V2) in Chinese patients with hypertension in primary care. Qual Life Res. 2019;28: 2851-7.

30. Thabane L, Ma J, Chu R, Cheng J, Ismaila A, Rios LP, et al. A tutorial on pilot studies: the what, why and how. BMC Med Res Methodol. 2010;10:1.

31. Soares I, Carneiro AV. Intention-to-treat analysis in clinical trials: principles and practical importance. Rev Port Cardiol. 2002;21:1191-8.

32. Redfern A, Peters S, Luo R, Cheng Y, Li C, Wang J, et al. Sex differences in the awareness, treatment, and control of hypertension in China: a systematic review with meta-analyses. Hypertens Res. 2019;42:273-83.

33. Fan M, Yu C, Guo Y, Bian Z, Li X, Yang L, et al. Effect of total, domainspecific, and intensity-specific physical activity on all-cause and cardiovascular mortality among hypertensive adults in China. J Hypertens. 2018;36:793-800.

34. Lodi E, Carollo A, Martinotti V, Modena MG. Hypertension and pharmacological therapy in women. High Blood Press Cardiovasc Prev. 2018:25:147-50.

35. Flachskampf FA, Gallasch J, Gefeller O, Gan J, Mao J, Pfahlberg AB, et al. Randomized trial of acupuncture to lower blood pressure. Circulation. 2007; 115:3121-9

36. Chen $H$, Shen FE, Tan XD, Jiang WB, Gu YH. Efficacy and safety of acupuncture for essential hypertension: a meta-analysis. Med Sci Monit. 2018:24:2946-69.

37. Cai H, Zhou Q, Bao G, Kong X, Gong LY. Transcutaneous electrical nerve stimulation of acupuncture points enhances therapeutic effects of oral lactulose solution on opioid-induced constipation. J Int Med Res. 2019;47: 6337-48.

38. Hsu YC, Liang IT, Huang SY, Wang HS, Soong YK, Chang CL. Transcutaneous electrical acupoint stimulation (TEAS) treatment improves pregnancy rate and implantation rate in patients with implantation failure. Taiwan J Obstet Gynecol. 2017;56:672-6.

39. Hadadian F, Sohrabi N, Farokhpayam M, Farokhpayam H, Towhidi F, Fayazi $\mathrm{S}$, et al. The effects of Transcutaneous Electrical Acupoint Stimulation (TEAS) on fatigue in haemodialysis patients. J Clin Diagn Res. 2016;10:YC0101 YC04.

40. Bolbrinker J, Zaidi Touis L, Gohlke H, Weisser B, Kreutz R. European guidelines on lifestyle changes for management of hypertension: awareness and implementation of recommendations among German and European physicians. Herz. 2018;43:352-8.

\section{Publisher's Note}

Springer Nature remains neutral with regard to jurisdictional claims in published maps and institutional affiliations. 